Chekired, M.; Roubtsova, V.

2-D Pore-Particle Scale Model of the Erosion at the Boundary of Two Soils Under Horizontal Groundwater Flow

Verfügbar unter / Available at:

https://hdl.handle.net/20.500.11970/100267

Vorgeschlagene Zitierweise / Suggested citation:

Chekired, M.; Roubtsova, V. (2010): 2-D Pore-Particle Scale Model of the Erosion at the Boundary of Two Soils Under Horizontal Groundwater Flow. In: Burns, Susan E.; Bhatia, Shobha K.; Avila, Catherine M. C.; Hunt, Beatrice E. (Hg.): Proceedings 5th International Conference on Scour and Erosion (ICSE-5), November 7-10, 2010, San Francisco, USA. Reston, Va.: American Society of Civil Engineers. S. 550-559. 


\title{
2-D Pore-Particle Scale Model of the Erosion at the Boundary of Two Soils Under Horizontal Groundwater Flow
}

\author{
M. Chekired ${ }^{1}$ and V. Roubtsova ${ }^{1}$ \\ ${ }^{1}$ Institut de recherche d'Hydro-Quebec, Expertise Robotique et Civil, 1740 boul. \\ Lionel-Boulet, Varennes (Quebec) J3X 1S1
}

\section{ABSTRACT}

Modeling particle-fluid systems in porous media encountered in many scientific and engineering applications presents a significant challenge. This paper outlines a hydrodynamic flow at a low Reynolds number through saturated porous media which is generated virtually using only the grain-size distribution curves of soils. In order to represent the pore and granular structures of soils, a novel model was developed using a fractal approach. For a given porosity, particle and pore size distributions were successfully modeled for a wide range of soils. We tested the model's conductivity behaviour by carrying out a steady-state flow through a fractal structure. Soil particle movement was modeled using the discrete element method (DEM), and the hydrodynamic flow was simulated by the well-known marker-andcells (MAC) method, with special conditions imposed at the particle boundaries. The fluid-particle interaction was taken into account in our calculations using the method of direct force integration at the surface of particles. Several comparisons of our numerical results to those of published experiments for particle-particle and particlewall interaction in a viscous fluid show very good agreement. Finally, an example of a possible erosion scenario at the interface of two different soils under a horizontal flow is presented. The results and a discussion of this model's applicability are also presented. This study is a part of an extensive program which includes 3-D simulations aimed at gaining a better understanding erosion phenomena in soils made up of irregularly shaped particles under hydrodynamic flow.

\section{Introduction}

Microscopic numerical analysis can be an effective tool to study the phenomenon of internal erosion. Pore-scale models of viscous fluid flow based on the Navier-Stokes equations take in account such details as the real water velocity and the water pressure in the pore channels. With this flow pattern, it is possible to calculate the real drag force, which is a primary factor determining the erosion threshold. The interaction between soil particles is integrated into the model.

\section{Fractal scaling of soil particle-size distributions}

A detailed study of the pore solid fractal (PSF) model was conducted by Perriers et al. (1999). The generation of a PSF model starts with the initiator which consists of a square of length $L$ in 2-D. This square is divided into $R^{2}$ sub squares with length $L / R, R$ being the scaling factor. The sub squares present the pores $(P)$, particles (S), and unspecified elements $\left(F=R^{2}-P-S\right)$ that will be devised in the next iteration step. All unspecified elements will be assigned to pores for the last iteration. 
This model was extended by combining three fractal processes in series (3GPSF) by Lehmann et al. (2003). Each generator was applied for a certain number of iterations. A more realistic model is obtained by changing the solid squares to solid round particles (see Figure 1).

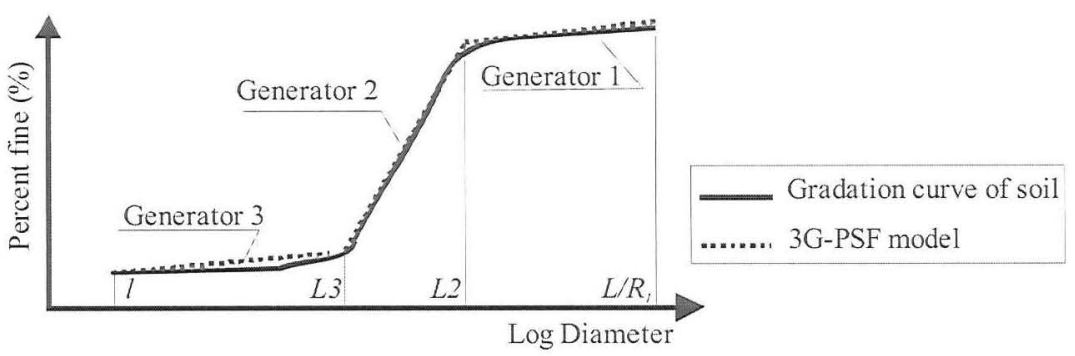

Generator 1

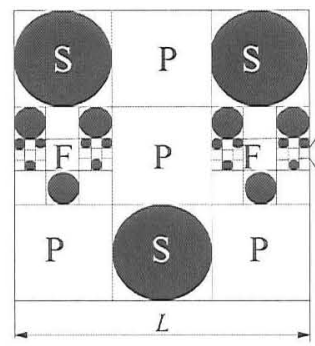

Generator 2

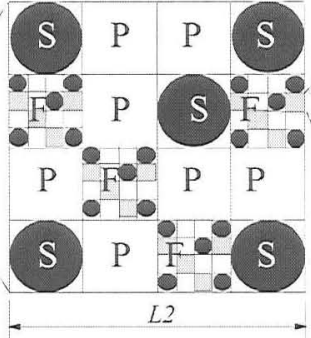

Generator 3

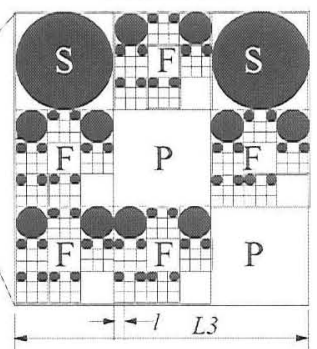

Figure 1. Construction of a $3 \mathrm{G}-\mathrm{PSF}$ with round particles.

For the first generator: $R=3, S=3, P=4$, and $n=3$; for the second generator: $R=4$, $S=5, P=7$, and $n=2$; and for the third generator: $R=3, S=2, P=2$, and $n=3$.

After a number of iterations, the porosity is found as follows:

$$
\begin{aligned}
& \phi_{n 1+n 2+n 3}=\frac{P_{1}+(1-0.25 \times \pi) S_{1}}{P_{1}+S_{1}}\left(1-\left(\frac{F_{1}}{R_{1}^{2}}\right)^{n 1}\right)+\left(\frac{F_{1}}{R_{1}^{2}}\right)^{n 1} \frac{P_{2}+(1-0.25 \times \pi) S_{2}}{P_{2}+S_{2}}\left(1-\left(\frac{F_{2}}{R_{2}^{2}}\right)^{n 2}\right) \\
& +\left(\frac{F_{1}}{R_{1}^{2}}\right)^{n 1}\left(\frac{F_{2}}{R_{2}^{2}}\right)^{n 2} \frac{P_{3}+(1-0.25 \times \pi) S_{3}}{P_{3}+S_{3}}\left(1-\left(\frac{F_{3}}{R_{3}^{2}}\right)^{n 3-1}\right)+\left(\frac{F_{3}}{R_{3}^{2}}\right)^{n 3-1}\left(\frac{P_{3}+F_{3}+(1-0.25 \times \pi) S_{3}}{R_{3}^{2}}\right)
\end{aligned}
$$

where the indices 1,2 , and 3 correspond to the first, second, and third generators respectively. $n$ is the number of iterations.

The best approximation of gradation curves associated with a given porosity determines the parameters of the generators. A specific algorithm dedicated to ensuring a flow path was used.

Different particle locations can be generated randomly with the same parameters of 3G-PSF model. A comparison of the particle size distribution of various soil samples with those obtained by fractal modeling is shown in Figure 2. 


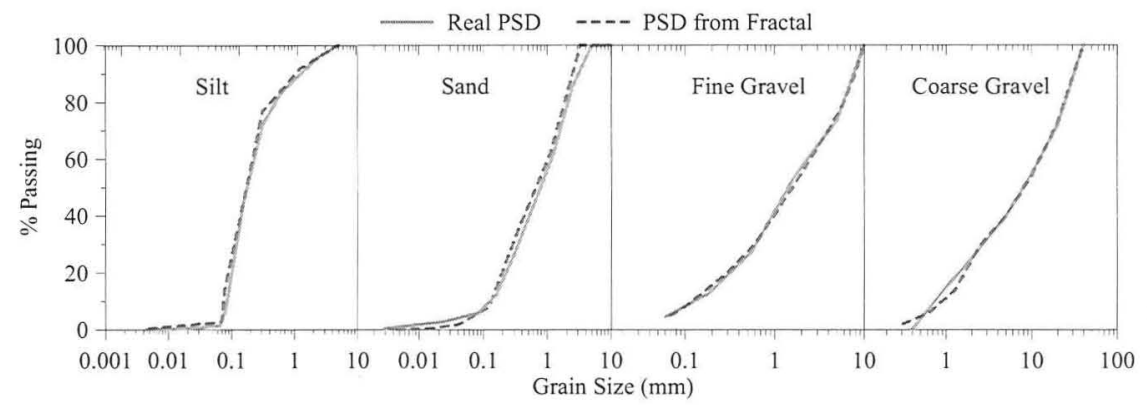

Figure 2. Comparison of PSD for various soil samples with those obtained from the fractal model.

\section{Flow through generated soils}

The water flow through a pore channel is described by the Navier-Stokes equations for viscous-incompressible liquids, and is expressed by:

$$
\begin{aligned}
& \rho\left[\frac{\partial \vec{v}}{\partial t}+(\vec{v} \nabla) \vec{v}\right]=-\nabla p+\eta \nabla^{2} \vec{v}+\vec{f} \\
& \nabla \vec{v}=0
\end{aligned}
$$

where $\rho$ is the density of the liquid $\left(\mathrm{kg} / \mathrm{m}^{3}\right), t$ is the time (s), $\vec{v}$ is the flow velocity $(\mathrm{m} / \mathrm{s}), p$ is the water pressure $(\mathrm{Pa}), \eta$ is the dynamic viscosity $(\mathrm{Pa} \mathrm{s})$, and $f$ is the force of gravity $(\mathrm{N})$.

We chose the well-known marker-and-cell (MAC) method to solve this system of equations (Harlow and Welch (1965)). (1963))

The coefficient of permeability $(\mathrm{m} / \mathrm{s})$ for this soil sample is (Scheidegger

$$
K=-\frac{\langle v\rangle s \rho g}{\Delta p}
$$

where $\langle v\rangle$ is the average velocity in the soil $(\mathrm{m} / \mathrm{s}), s$ is the length of the sample in the direction of flow (m), and $\Delta p$ is the pressure difference at the boundary $(\mathrm{Pa})$.

The hydrodynamic flow through four different soil samples generated by the fractal model is presented in Figure 3. Comparison of porosity and the coefficient of permeability calculated from the fractal model and from the empirical formula for different soils is presented in Tables 1 and 2 respectively.

The empirical porosity of the soil was calculated by $\phi=0.255\left(1+0.83^{U}\right)$ (Vukovic and al. (1992)), where $U=d_{60} / d_{10}$ is the coefficient of grain uniformity, $d_{60}$ and $d_{10}$ represent the grain diameter in $\mathrm{mm}$ at which $60 \%$ and $10 \%$ grains are finer respectively. The porosity of the fractal model was calculated using equation 1 . 


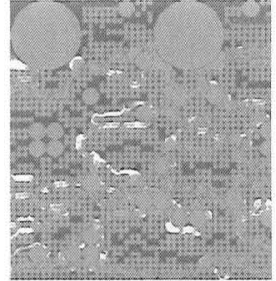

Silt

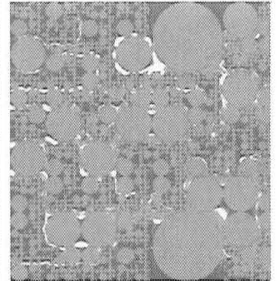

Sand

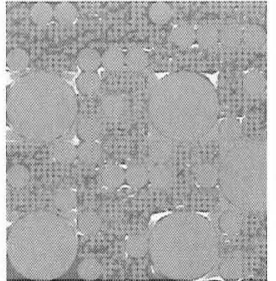

Fine gravel

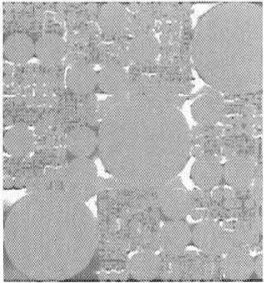

Coarse gravel

Low flow velocity

High flow velocity

Figure 3. Hydrodynamic flow through generated soils

Table 1. Comparison of porosity

\begin{tabular}{l|c|c|c|c}
\hline & Silt & Sand & Fine Gravel & Coarse Gravel \\
\hline Empirical & 0.406 & 0.303 & 0.261 & 0.268 \\
\hline Fractal & 0.405 & 0.314 & 0.265 & 0.279 \\
\hline
\end{tabular}

Table 2. Comparison of coefficient of permeability $(\mathrm{m} / \mathrm{s})$

\begin{tabular}{ll|c|c|c|c}
\hline & Silt & Sand & Fine Gravel & Coarse Gravel \\
\hline \multirow{2}{*}{ Empirical } & $1.07 \cdot 10^{-04}$ & $2.03 \cdot 10^{-04}$ & $2.17 \cdot 10^{-04}$ & $5.09 \cdot 10^{-03}$ \\
\hline \multirow{2}{*}{ Fractal } & Min & $1.61 \cdot 10^{-04}$ & $2.18 \cdot 10^{-04}$ & $8.61 \cdot 10^{-05}$ & $9.68 \cdot 10^{-04}$ \\
& Max & $1.94 \cdot 10^{-04}$ & $2.65 \cdot 10^{-04}$ & $3.97 \cdot 10^{-04}$ & $3.44 \cdot 10^{-03}$ \\
\hline
\end{tabular}

The empirical coefficient of permeability was calculated using the Pavchich formula (Goldin and Rasskazov (1992)), expressed as follows:

$$
K=\frac{g}{v} 4 \cdot 10^{-3} B \sqrt[3]{U} \frac{\phi^{3}}{(1-\phi)^{2}} d_{17}^{2}
$$

where $g$ is the acceleration due to gravity, $v$ is the kinematic viscosity of water $\left(\mathrm{m}^{2} / \mathrm{s}\right)$, $B$ is equal to 1 for sand and gravel and $0.35-0.4$ for pebbles, and $d_{17}$ represents the grain diameter in $\mathrm{mm}$ for which $17 \%$ of the sample are fine then. This formula has been found to be satisfactory for a wide range of soils. We present the extreme (Min. and Max.) values for the coefficient of permeability calculated during simulations for various particle locations. Knowing that the permeability is a $3 \mathrm{D}$ property, the permeability calculated using a $2 \mathrm{D}$ concept is not fully conclusive for all soils selected in this study.

\section{Motion and interaction of particles in fluids (DEM)}

The movement of granular particles is modeled using the discrete element method (DEM) proposed by Cundall and Strack (1979). The motions of particle $i$ caused by its interactions with neighboring particles is described by the equations:

$$
m_{i} \frac{d \vec{V}_{i}}{d t}=m_{i} \vec{g}+\sum_{j=1}^{k}\left(\vec{F}_{c n, i j}+\vec{F}_{d n, i j}+\vec{F}_{c t, i j}+\vec{F}_{d t, i j}\right)+\vec{F}_{f l}
$$


where:

$$
I_{i} \frac{d \vec{\omega}_{i}}{d t}=\sum_{j=1}^{k}\left(\vec{T}_{i j}+\vec{M}_{i j}\right)+\vec{M}_{f l}
$$

$m_{i}, I_{i}$, are the mass $(\mathrm{kg})$ and moment of inertia $\left(\mathrm{kgm}^{2}\right)$ of particle $i$ respectively $\vec{V}_{i}$ and $\vec{\omega}_{i}$ are the translational $(\mathrm{m} / \mathrm{s})$ and rotational $\left(\mathrm{s}^{-1}\right)$ velocities of particle $i$ $k$ is the number of neighboring particles.

$\vec{F}_{c n, i j}=-K_{n} \delta_{n}^{\frac{3}{2}} \vec{n}$ is the normal force of contact (N)

$\vec{F}_{d n, i j}=-C_{n} \vec{V}_{n, i j}$ is the normal damping force (N)

$\frac{d \vec{F}_{c t, i j}}{\vec{d} \delta_{t}}=-K_{t}$ is the tangential force of contact (N). The value of this force is

limited by $\left|F_{c t, i j}\right| \leq \mu_{s} F_{c n, i j}$

$\vec{F}_{d t, i j}=-C_{t} \vec{V}_{t, i j}$ is the tangential damping force (N)

$\vec{F}_{f l}$ is the drag force (N)

$\vec{T}_{i j}=R_{i}\left(\vec{F}_{c t, i j}+\vec{F}_{d t, i j}\right)$ is the torque moment (N'm)

$\vec{M}_{i j}=-\mu_{r}\left|\vec{F}_{c n . i j}\right| \frac{\vec{\omega}_{i}}{\left|\vec{\omega}_{i}\right|}$ is the friction torque (N'm)

$\vec{M}_{f l}$ is the drag moment (N.m)

$$
K_{n}=\frac{4}{3} E^{*} \sqrt{R^{*}}, C_{n}=2 \sqrt{m^{*} K_{n}}, K_{t}=2 \sqrt{R \delta_{n}}\left(\frac{G_{i}}{2-v_{i}}+\frac{G_{j}}{2-v_{j}}\right), C_{t}=K_{n} \sqrt{\frac{K_{t}}{K_{n}}}
$$

$\delta_{n}, \delta_{t}$ are the normal and tangential contact displacement respectively:

$$
\frac{1}{E^{*}}=\frac{1-v_{i}^{2}}{E_{i}}+\frac{1-v_{j}^{2}}{E_{j}} \text { and } \frac{1}{R^{*}}=\frac{1}{\left|R_{i}\right|}+\frac{1}{\left|R_{j}\right|}
$$

where $E$ is Young's modulus $(\mathrm{Pa}), v$ is the Poisson ratio, $R_{i}$ is the particle radius $(\mathrm{m})$, and $\mu_{s}, \mu_{r}$ are the sliding and rolling $(\mathrm{m})$ friction coefficients respectively. The determination of interaction forces has been detailed by Zhou et al. (2002) and Nakashima (2004).

To obtain the proper particle-liquid interaction, we need to integrate the stress tensor over the particle surface. Currently, we use a 2-D model for the water flow and the spherical particles that move and rotate in a 2-D plane. First, we integrate the stress tensor over the particle surface (circle) at the plane of flow.

$$
\left.\vec{F}_{f l}=\oint_{A}\left(-p+\eta \mid \nabla \vec{v}+(\nabla \vec{v})^{t}\right]\right) \hat{n} d A
$$

where $A$ is the particle outline projection $(\mathrm{m})$ and $\hat{n}$ is the normal to the circular particle.

This force acts per unit length around infinite cylinder into flow. The standard curves for a drag coefficient of a cylinder and a sphere (Clift et al. (1978)) are shown 
in Figure 4. For comparison, we have also plotted the Stokes curve. For a low Reynolds number, we can see that

$$
C_{D s} \approx 1.5 \cdot C_{D c}
$$

where $C_{D s}$ and $C_{D c}$ are the drag coefficients for a sphere and a cylinder respectively.

Therefore, the relationship of the drag force for the cylinder and the sphere under the same flow conditions can be rewritten as:

$$
\frac{F_{s}}{F_{c}}=\frac{3 \pi d}{8}
$$

where $F_{s}$ is the drag force for the sphere $(\mathrm{N}), F_{c}$ is the drag force for the cylinder $(\mathrm{N} / \mathrm{m})$, and $\mathrm{d}$ is the diameter of the circular particle $(\mathrm{m})$. The numerical results obtained for particles 0.5 and $1 \mathrm{~mm}$ in diameter are shown in Figure 4.

The buoyancy force is included in formula 4 , and is adjusted for a spherical particle. The no-slip liquid boundary condition on each particle surface was also implemented. We used the condition proposed by Kalthoff et al. (1997). All the liquid grid velocities located "inside" a particle are constrained to equal the particle velocity before the evaluation of the temporal velocity at each time step.

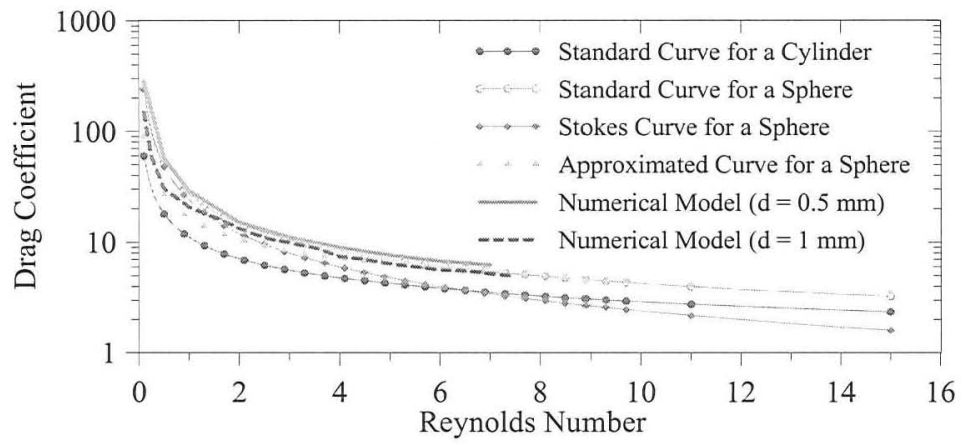

Figure 4. Drag coefficient as a function of the Reynolds number.

\section{Results validation}

To validate the numerical results obtained, experimental data obtained by Zhang et al. (1999) were selected. These data concern the settling of spherical particles and the dynamic behavior of their collision in a viscous fluid. Several experiments were carried out with different particle densities and dynamic viscosities. Lubrication theory predicts that contact is impossible at low Reynolds numbers. However, due to the existence of microscopic surface roughness on real particles, direct particle-particle contact is possible. Several studies on the rebounding of colliding particles have been published in recent years. We use an algorithm in which the collision process starts when the separation between particles is less than one MAC cell. The change in velocity with distance for spherical particles before and after colliding with a fixed particle in aqueous glycerine is shown in Figure 5(a) and 
5(b) respectively. Figure 5(b) concerns only the example with a particle density of $2180 \mathrm{~kg} / \mathrm{m}^{3}$ and fluid with a dynamic viscosity of $0.053 \mathrm{~Pa}$ s. Our results are depicted in Figure 6. The visualization allows users to explore relationships in the data, and to better present the results to scientists and engineers. In this way, particle-tracking models can be better integrated into decision support systems.
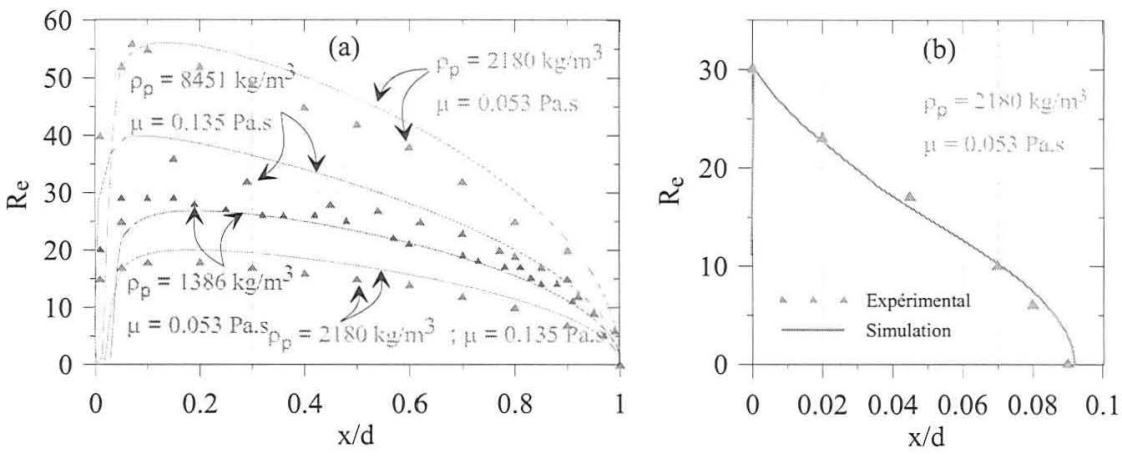

Figure 5. Comparison of simulated and experimental data for colliding particles in viscous fluid
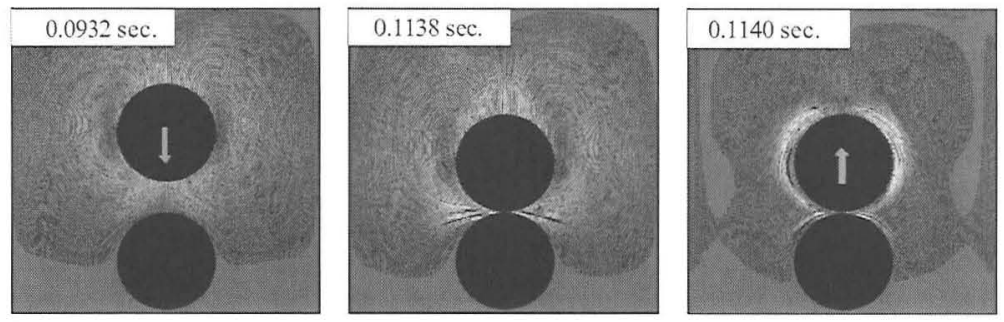

Figure 6. Visualization of the velocity field induced by the normal collision of settling particle with fixed particle

Figure 7(a) compares the numerical data and experimental data for the settling of glass beads and also after they collide with a wall in water. A schematic representation of the test setup is shown in Figure 7(b). Computational visualization of the flow streamlines during settling of the glass beads and as they approach the wall is shown in Figure 8.

Another validation example concerns the trajectories of a particle for the oblique collision of two glass beads. Comparison of the experimental and simulation results is presented in Figure 9(a). Visualization of the flow streamlines before and after collision is presented in Figure 9(b). We have also compared the results obtained by our model with other experimental results (Zhao and Davis (2002), Bouard and Coutanceau (1986)), and found that they agree very well. 

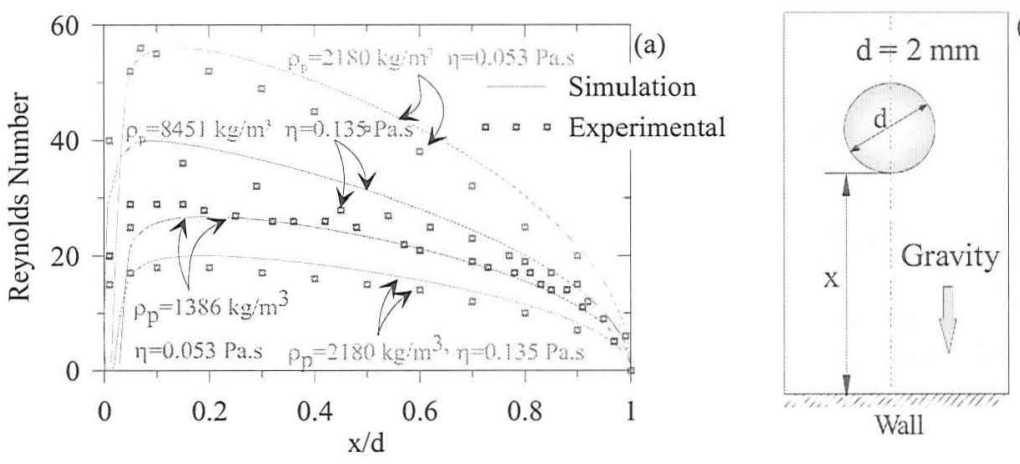

(b)

Figure 7. (a) Comparison of the simulation and experimental data for the collision of a glass bead with a wall in water, and (b) Experimental setup
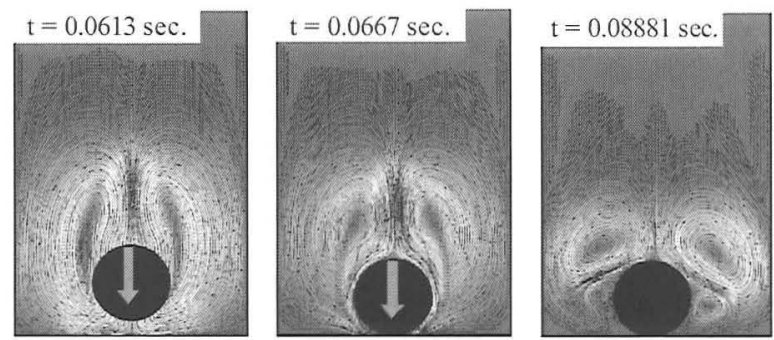

Figure 8. Visualization of the flow streamlines induced by collision of the particle with the wall

\section{Simulation of erosion at the interface of two soils}

In simulating a possible erosion scenario in 2-D between two layers of different soils generated virtually, special conditions were imposed: the top layer of soil particles (coarse gravel) was kept fixed at all times, while the bottom layer of soil particles (silt) was fixed until a steady-state flow was achieved. This allowed the silt particles to migrate under the effect of hydrodynamic forces. This approach was adopted to allow water to pass between the particles. These conditions would not be needed for a 3-D model.

Our objective with this example is to illustrate the potential of our model to examine the stability of a given base soil-filter system with a flow parallel to the contact interface. We simulate only a very small arrangement of soil particles containing 614 moving particles for silt and 14 fixed particles for coarse gravel. The density of the silt particles is $2700 \mathrm{~kg} / \mathrm{m}^{3}$. The size of the silt particles is $9.77 \cdot 10^{-3}$ $0.15 \mathrm{~mm}$ and that of the gravel particles is $1.11-0.278 \mathrm{~mm}$. The gradient is constant, and is equal to 0.063 . The stages of particle movement at different times are presented in Figure 10. The velocity of the water in gravel is greater than it is in silt, because the pore channels are larger in the gravel. As the fine particles begin to leave 
the soil, the pores become larger and the channels straighter. Increasing flow velocity subsequently causes major silt particle migration.

(a)

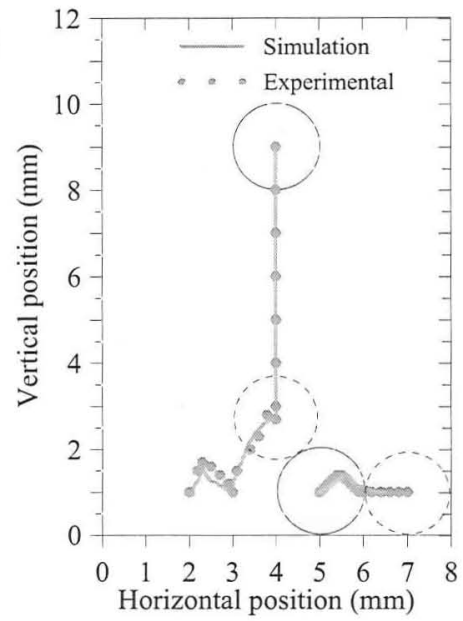

(b)

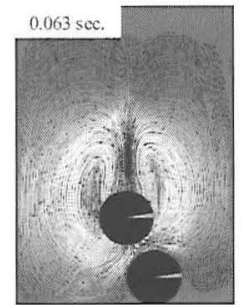

$0.08+\mathrm{sec}$

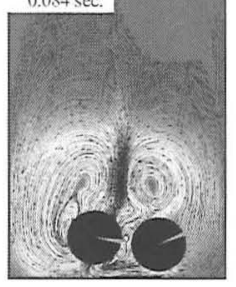

$0.068 \mathrm{sec}$.

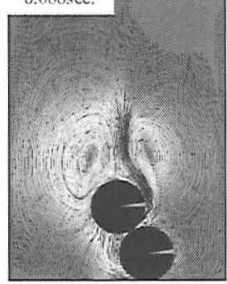

$0.089 \mathrm{sec}$.

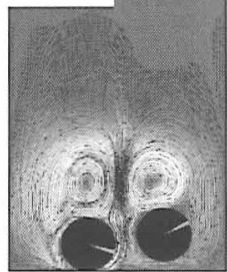

Figure 9. (a) Experimental and simulation results of the trajectories of two particles colliding, and (b) Visualization of results
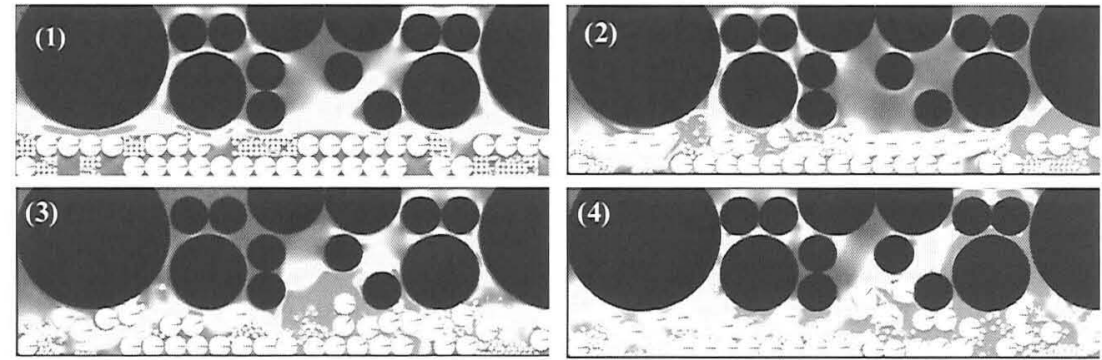

Figure 10. Visualization of the erosion process: (1) steady-state flow with fixed particles $(t=0.00 \mathrm{sec}$.), (2) Motion of the finest particles $(t=0.013 \mathrm{sec}$.),

(3) Motion of the largest particles ( $t=0.022 \mathrm{sec}$.), and (4) Erosion ( $t=0.030 \mathrm{sec}$.))

\section{Conclusion}

Our preliminary results in this paper indicate that the proposed 2-D model can virtually generate different kinds of soils successfully using only grain size distribution curves, and can estimate soil characteristics, such as the coefficient of permeability. The simulation results of the dynamic behavior of particles in motion in a fluid imply that the model can precisely describe the hydrodynamic behavior of particles in motion, predicting the motion of the particles before collision, the collision between particles, the rebounding of particles following contact, and the motion of particles after contact. Several other simulations show that the model 
results agree very well with those obtained from experiments. An example of the visualization of erosion at the contact interface between two different soils under horizontal flow shows the capability of the model. It can determine the transport and deposition rates of particles, where the porous media were subjected to hydrodynamic flow, and it can simulate the microscopic features of the process of internal erosion. Currently, the model has some limitations, such as the round shape of the particles and the 2-D water flow. Based on the verification work presented in this paper, the focus will now move to the development of 3-D model, which can accommodate the real shape of natural particles and include the compaction effects and cohesive forces.

\section{REFERENCES}

Bouard, R. and Coutanceau, M. (1986). "Étude théorique et expérimentale de l'écoulement engendré par un cylindre en translation uniforme dans un fluide visqueux en régime de Stokes." Journal of Applied Mathematics and Physics (ZAMP), Vol. 37 (5): 673-684.

Clift, R., Grace, J. R., and Weber, M. E. (1978). Bubbles, Drops and Particles, Academic Press, New York, 380 p.

Cundall, P. and Strack, O. (1979). "A Discrete numerical model for granular assemblies." Geotechnique, Vol. 29(1): 47-65.

Goldin, A. L. and Rasskazov, L. N. (1992). Earth Dam Design, Geotechnika 2. Balkema, Rotterdam, 328 p.

Harlow, F. H. and Welch J. E. (1965). "Numerical calculation of time-dependent viscous incompressible flow of fluid with a free surface," The Physics of Fluids, Vol. 8: 2182-2189.

Kalthoff, W., Schawarzer, S., and Herrmann, H. J. (1997). "Algorithm for the simulation of particle suspensions with inertia effects," Physical Review, Vol. 56(2): 2234-2242.

Lehmann, P., Stähli, M., Papritz, A., Gygi, A., and Flühler, H. (2003). "A Fractal Approach to Model Soil Structure and to calculate thermal conductivity of soils," Transport in porous media, Vol. 52: 313-332.

Nakashima, H. (2004). "Discrete Element Method (DEM) and its possible application." SICE Annual Conference in Sapporo, Vol. 2: 1104-1107.

Perrier, E., Bird, N., and Rieu, M. (1999). "Generalizing the fractal model of soil structure: the pore-solid fractal approach." Geoderma, Vol. 88: 137-164.

Scheidegger, A. E. (1963). "The Physics of Flow Through Porous Media." University of Toronto Press, Toronto.

Vukovic, M. and Soro, A. (1992). "Determination of Hydraulic Conductivity of Porous Media from Grain-Size Composition." Water Resources Publications, Littleton, Colorado, CO, 83 p.

Zhang, J., Fan, L.-S., Zhu, C., Pfeffer, R., and Qi, D. (1999). "Dynamic behavior of collision of elastic spheres in viscous fluids." Powder Technology, Vol. 106: 98-109.

Zhao, Y. and Davis R. H. (2002) "Interaction of two touching spheres in a viscous fluid." Chemical Engineering Science, Vol. 57: 1997-2006.

Zhou, Y. C., Xu, B. H., Yu, A. B., and Zulli, P. (2002). "An experimental and numerical study of the angle of repose of coarse spheres." Powder Technology, Vol. 125: 45-54. 